\title{
LA AMBIVALENCIA COMO CATEGORÍA SOCIOLÓGICA EN SIMMEL
}

\section{Fernando Robles}

Universidad de Concepción, Chile

\begin{abstract}
RESUMEN
En este trabajo se destaca la trascendencia de la sociología de Georg Simmel para la tematización del sujeto de la modernidad en la microsociología contemporánea. A partir de la matriz de interacción configurada por el cruce de los círculos sociales, se analiza la actualidad de Simmel y su análisis de la moda, en el contexto delimitado por la conformación de las ambigüedades de la individualización y la colectivización de la identidad de los sujetos modernos. A continuación, la dominación de la cultura objetiva sobre la producción subjetiva, uno de los teoremas elementales de la sociología simmeliana, sirve de pivote para tematizar el tránsito de la autonomización de lo objetivo hacia la individuación de la cotidianeidad del sujeto de la modernidad.
\end{abstract}

«Ser modernos es encontrarnos en un entorno que nos promete aventuras, poder, alegría, crecimiento, transformación de nosotros y del mundo y que, al mismo tiempo, amenaza con destruir todo lo que tenemos, todo lo que sabemos, todo lo que somos.»

Marshall BERMAN

La designación microsociología fue introducida, aunque con connotaciones completamente diferentes, por George Gurvitch ${ }^{1}$ y Jacob Moreno para designar una esfera específica de la teoría sociológica que se propone explicar fenómenos

1 George Gurvitch (1962): Tratado de Sociología, tomo I, Losada, Buenos Aires. 
y procesos sociales analizando las unidades más pequeñas de la sociedad, comprendidos en la interacción directa de individuos y en su dependencia respecto de las estructuras sociales que componen su entorno. Uno de los precursores elementales de la microsociología en el ámbito de la sociología de la comprensión es Georg Simmel (1858-1918), «el primer sociólogo de la modernidad»², contemporáneo de Weber y denominado el fundador de la llamada sociología formal, de acuerdo a la cual el objeto fundamental de la sociología debiese ser el estudio de las formas histórico-culturales y constantes de aculturación social de las sociedades, entendidas como un proceso multifacético de intercambio material e inmaterial entre sujetos sociales, desde los cuales arranca en última instancia el sentido real de lo social y sus instituciones. Ella insiste por ello en la importancia de las formas, de los estilos, de las figuras, de las configuraciones que los procesos de intercambio cultural contienen y dan lugar, antes que fijarse en la búsqueda de las esencias; de allí que, en el contexto de su inspiración evidentemente neokantiana, la sociología de Simmel sea simultáneamente la negación de la metafísica. Sin caer en la desontologización total de la microsociología contemporánea, la sociología de Simmel inaugura un ámbito de reflexión completamente nuevo en la tematización de la modernidad.

En efecto, superando por un sendero diferente la alternativa antiontológica que con posterioridad formulara Heidegger ${ }^{3}$, Simmel esboza una sociología pluralista y relativista de las formas, donde se imposibilita la postulación de un único mundo significativo, y se afirma «más bien una pluralidad de mundos, donde ninguno puede reclamar para sí la primacía ontológica o epistemológica sobre los demás» ${ }^{4}$ y que, siendo una de las interpretaciones más peculiares (probablemente la más heterodoxa hasta la obra radical de Herbert Blumer ${ }^{5}$ ) que se haya realizado de Kant, advierte de principio a fin sobre la esterilidad del reduccionismo sociológico: para Simmel es imposible abarcar la realidad en su integridad inmediata, sino únicamente observarla desde diversos ángulos por separado, constituyendo una pluralidad de objetos investigativos relativamente independientes los unos de los otros. «De lo que se trata es, ente todo, de esto: que el contenido social de la vida, aunque pueda ser explicado totalmente por los antecedentes sociales y por las relaciones sociales mutuas, debe considerarse al propio tiempo también, bajo la categoría de la vida individual, como vivencia del individuo u orientado enteramente hacia el individuo... La sociedad consta de seres que por un lado se sienten cual existencias plenamente sociales y, por otro lado, cual existencias personales sin cambiar por ello su contenido» ${ }^{6}$.

2 David Frisby (1988): «Georg Simmel: Primer sociólogo de la modernidad», en Josep Picó (ed.) (1988): Modernidad y Posmodernidad, Alianza, Madrid, pp. 51-86.

H. Dreyfus (1996): Ser-en-el-Mundo, Cuatro Vientos, Santiago.

${ }^{4}$ Antonio Gil Villegas (1997): «El fundamento filosófico de la teoría de la modernidad en Simmel», en Estudios Sociológicos, XV: 43, México, p. 28.

${ }^{5}$ H. Blumer (1982): Interaccionismo Simbólico, Hora, Barcelona.

${ }^{6}$ Georg Simmel (1986): Sociología II, Alianza, Madrid, pp. 50-51. 
Probablemente porque Simmel no únicamente proclamara ámbitos cada vez más amplios de libertad individual para sus contemporáneos, sino que reclamara esa misma libertad en sus trabajos sociológicos, es que no fue tampoco proclive a entregar definiciones explícitas de las categorías con las que opera. De allí que la evasividad con que se niega a formular conceptos acabados se vea aparejada a una verdadera obsesión por la ejemplificación concreta del significado práctico de su metodología; su obra está salpicada de elaboraciones sutiles, detalladas y profundas de fenómenos específicos (aunque no aislados) como la moda, la coquetería o lo femenino, integrando manifestaciones históricas del desenvolvimiento de la cultura (como el dinero) a la construcción de los significados, por ejemplo, en la esfera de lo estético. La obra de Simmel se caracteriza por la negación de lo unicéntrico y, consecuentemente, sus trabajos inauguran en la práctica el análisis de uno de los fenómenos tanto fascinantes como esenciales de la modernidad post-tradicional: la ambivalencia y la peculiaridad policéntrica de las sociedades contemporáneas ${ }^{7}$. Pero al mismo tiempo que no resulta exagerado indicar que el mismo Simmel se situó en el centro de los «círculos sociales» de la cultura occidental y que su obra influyó a la mayor parte de la sociología contemporánea, abarcando desde Weber, Sombart y Mannheim hasta Luckács y Bloch, no es superfluo tampoco recordar que su vida no se eximió de los apremios y vicisitudes propios de su condición de judío y socialista, más aún, de un intelectual lleno de originalidad y blanco de las envidias e intrigas propias del ambiente académico willhelmino: su doctorado fue reprobado, y si bien su hogar intelectual fue siempre Berlín, allí jamás salió de la insegura categoría de Privatdozent, obteniendo al fin de sus días un puesto de profesor titular en Estrasburgo, una ciudad entonces puramente provinciana ${ }^{8}$.

Siendo múltiples los trabajos de Simmel posibles de cobijar dentro de su microsociología, vamos a destacar el tema del cruce de los círculos sociales, la sociología de la moda en el contexto de la dialéctica entre cultura objetiva y cultura subjetiva, para mostrar de qué manera su sociología puede abrir camino a la autonomización de la cotidianeidad como una respuesta a la creciente autonomización de lo objetivo.

\section{EL CRUCE DE LOS CÍRCULOS SOCIALES}

Los sujetos sociales, así como sus formas de producción simbólica y material que de ellos resulta, no son el resultado de objetivaciones que puedan ser

7 Z. BAuman (1991): Modernity and Ambivalence, Polity Press, London; Niklas LuHMAnN (1997): Die Gesellschaft der Gesellschaft, Suhrkamp, Frankfurt a. M.; Ulrich BECK (1997a): «La reinvención de la política: hacia una teoría de la modernización reflexiva», en U. BECK, A. GIDDENS y S. LASH: Modernización reflexiva. Política, tradición y estética en el orden social moderno, Alianza, Madrid, pp. 13-74.

${ }^{8}$ David Frisby (1984): Georg Simmel, FCE, México; Jürgen Habermas (1988): «Simmel como intérprete de su época», en Georg Simmel (1988), pp. 273-285. 
concebidas como divorciadas del curso de su vida cotidiana, sino que son el resultado del cruce de círculos sociales, comprendido como la matriz de interacción real donde se desenvuelve la vida social. Así entonces, los grupos a los que el individuo pertenece conforman un sistema de coordenadas, de forma tal que la aparición de cada nuevo grupo determina al individuo de un modo más exacto e inequívoco. La pertenencia a cada uno de ellos deja abierto un campo inexplorado para la expansión de la individualidad, y cuanto mayor sea su número, menos probable será que existan otras personas que tengan la misma combinación de grupos y que estos círculos numerosos vuelvan a cruzarse en un punto. Por lo tanto, el punto de intersección de los círculos sociales es determinante para el sendero que siga la individualidad.

Hablando platónicamente, cada uno de los sujetos sociales participa de tantas ideas como cualidades posea, adquiriendo así su determinación incanjeable individual ${ }^{9}$. Mediante los diversos elementos constitutivos de la vida social en la cual nos desenvolvemos, formamos nuestra propia subjetividad y, por tanto, la personalidad en la cual se combinan individualmente los elementos de la cultura. Una vez que la síntesis de lo subjetivo ha podido dar origen a lo objetivo, la misma síntesis de lo subjetivo fecunda, a su vez, una subjetividad nueva y diferente; de la misma manera que la personalidad es capaz de entregarse al círculo social y hasta sumergirse en él para volver luego a reconquistar sus peculiaridades como resultado de este cruce de los círculos sociales que se verifica en cada persona ${ }^{10}$. Los círculos sociales de los que cada uno participa son elementos flexibles pero determinantes de las formas de asociación y socialización de los sujetos: «cuando, por ejemplo, la madre del marido interviene en una diferencia conyugal, sus instintos -en cuanto actúan, por decirlo así, a priori, y prescindiendo de todas las particularidades individuales del caso- - se inclinarán, unas veces al hijo, en virtud de los lazos de sangre, y otras veces a la nuera, como compañera de sexo»" ${ }^{11}$.

La conciencia social es el punto de coincidencia de incontables hilos sociales de significación práctica, que ajustan a su vez a la historia y a la biografía de los actores, y al mismo tiempo es la singularidad de sus formas de combinación. La intersección de estos círculos, lo que equivale a la situación concreta de la individualidad social, tiene una doble connotación: por un lado, es el fundamento de la integración simbólico-cultural de la subjetividad, en intercambio constante con las formas objetivas de aculturación histórica, y, por otro lado, es una fuente inextinguible de conflictos de diversa y variada índole "que amenazan al individuo con un dualismo espiritual, y hasta con íntimos desgarrones ${ }^{12}$; ahora bien, la doble connotación que Simmel postula no significa que la pertenencia a varios (y hasta contradictorios) círculos sociales deje

\footnotetext{
9 Georg Simmel (1986): Sociología II, Alianza, Madrid, p. 263.

${ }_{10}$ Op. cit., p. 436.

11 Op. cit., p. 430.

12 Op. cit., p. 427.
} 
de tener efectos que fortalezcan y afirmen la unidad personal. De tal manera que la determinación sociológica del individuo será tanto mayor cuanto que los círculos determinantes sean más contiguos y más concéntricos, de manera que los círculos que se van estrechando lentamente como la nación o la profesión específica de cada uno no señalarán a la persona en su lugar individual y especial, pues el más estrecho de ellos significa, por sí mismo, la pertenencia a los más amplios. Los círculos interaccionales son, entonces, de distintos niveles de generalidad, y no sólo pueden sino que deben pertenecer a diversos segmentos de la vida social. Es cierto que las asperezas que resultan de la existencia de una cantidad considerable de círculos sociales son considerables y costosas, en particular si éstos son concéntricos, pues precisamente su carácter unicéntrico, al margen de pertenecer a sociedades de baja complejidad y diferenciación, significa un escollo para el desenvolvimiento de la libertad individual.

En contraste, la vida en la modernidad contemporánea impone a los individuos la existencia de una gran cantidad de círculos sociales policéntricos (en el aspecto profesional, de la sociedad científica, respecto de las instituciones civiles y políticas, de la familia y sus roles, etc.), lo que, además de conllevar un aumento considerable de las formas de la sociabilidad, significa también nuevos y desconocidos peligros para la libertad individual. De allí que Simmel, lejos de ensalzar las ventajas de la modernidad, se ocupe particularmente de develar sus riesgos.

A juicio de Simmel, con la ayuda de la intersección de los círculos sociales, es posible edificar una serie de tipologías históricas, dejando resaltar la posibilidad de conflictos entre ellos; por ejemplo, en la situación de sujetos sociales que se sitúen en la intersección de dos grupos que sean opuestos el uno del otro: el matrimonio gentilicio de sociedades arcaicas, la existencia del sacerdocio católico, las organizaciones de las profesiones medievales, son ejemplos que ilustran estas situaciones peculiares. Mientras, por un lado, lo específico de la individualidad permanece garantizado por la manera específica en que los círculos se combinan, esta individualidad es constitutiva para los círculos sociales como tales, de la manera que «la sociedad nace de los individuos, pero el individuo nace de las sociedades ${ }^{13}$. Con ello, la dialéctica entre individuo y sociedad asume una condición ausente de metafísica y atada a la ejecución de prácticas de producción y reproducción del sentido en contextos de interacción.

Los círculos sociales se enmarcan en el contexto de la evolución de las sociedades. En efecto, la diferenciación y la división del trabajo constituyen sólo los aspectos cuantitativos de la historia y la evolución de las sociedades, mientras que los aspectos cualitativos están determinados por la particularidad de las relaciones humanas, en medio de los círculos sociales, de tal forma que la comprensión de la historia y de la evolución sociales y, consecuentemente, la construcción de las tipologías histórico-explicativas que las ciencias sociales

13 Op. cit., p. 454. 
elaboren deben integrar, a partir de la visión microsociológica y concreta de los círculos grupales, las distintas formas en que se evidencia la división del trabajo y la diferenciación estructural. Al informar acerca de las configuraciones concretas que la vida social asume e iluminar las diversas probabilidades de conflicto que para cada uno de los actores sociales existe, la intersección de los círculos sociales es un instrumento de análisis agudo y exacto, que anuncia la conocida ejecución cotidiana del «método de la interpretación documental», uno de los elementos constitutivos del paradigma interpretativo contemporáneo ${ }^{14}$.

La multiplicidad de temas que Simmel estudia, pero sobre todo la creatividad de sus ejemplificaciones, abarcan tanto las formas históricas que las interacciones entre grupos de asociación temporal revisten (como es el caso de la asociación por juventud o por vejez), así como las relaciones afectivas al interior de la familia (relaciones entre las nueras y las suegras) hasta la relación salarial entre patrones y obreros. A este respecto, Simmel se pregunta en qué medida la situación específicamente interaccional de los obreros determina la organización de la internacional obrera y de qué forma se conserva simultáneamente un concepto jurídico superior que hace posible la huelga general, que no se emprende para satisfacer los fines de un sindicato en particular, sino para lograr derechos políticos de los obreros en general. Simmel apunta que algo similar sucede cuando se asocian patrones de las más diversas ramas de la economía, donde el patrón de una rama específica de actividad económica, como tal, no siente ningún interés por las relaciones que pudiesen existir entre obreros y patrones de otra rama. Lo que la coalición busca es fortalecer al patrón en general, frente al obrero en general. La visión que Simmel en tal sentido desarrolla supera y corrige las miopías de esquemas de interpretación que permanecen prisioneros de cosmovisiones totalizantes (como aquella que postula la universalidad de la lucha entre las clases) y que se desvinculan, consciente o inconscientemente, de la realidad de la asociaciones sociales concretas que los mismos actores sociales realizan. Precisamente ésta es una línea trabajo y de argumentación que se desplaza a lo largo y ancho de la obra simmeliana, desde la Filosofía del dinero ${ }^{15}$ hasta sus trabajos sobre estética. En tal sentido, Simmel argumenta que si bien es cierto que, en el marco de los conflictos de clase, la organización obrera es sincrónicamente lógica a la organización patronal, existen diferencias cualitativas elementales, y esto por tres razones. En primer lugar, porque los patrones son una minoría respecto de los obreros, lo que impide contraponerlos como si se tratara de equivalentes - lo que podemos determinar como una dimensión cuantitativa de análisis-. En segundo lugar, porque entre los patrones existe una necesidad estructural de competencia que

${ }^{14}$ H. GarfinKel y H. SACKS (1976): «Über formale Strukturen praktischer Handlungen», en Elmar Weingarten y Fritz Sack (eds.): Ethomethodologie. Beiträge zu eine Soziologie des Alltagshandelns, Suhrkamp, Frankfurt a.M., pp. 130-178.

${ }^{15}$ G. Simmel (1900): Die Philosophie des Geldes, Dunker und Humbolt Verlag, Berlín. 
entre los obreros no existe, al menos con la misma intensidad y con un contenido comparable - dimensión cualitativa-. En tercer lugar, por la existencia de las múltiples fusiones, alianzas y conflictos existentes en la estructura de cada una de las economías capitalistas - dimensión estructural ${ }^{16}$.

Otro ámbito de reflexión examinado por Simmel respecto de la relevancia de los círculos sociales se refiere a la condición social de la mujer en los albores del siglo XX. Pues hablando en general, así Simmel, lo que une a la mujer y la caracteriza es un concepto demasiado amplio - por ejemplo, las actividades propias en el sentido tradicional de la palabra- y consiste en aquello que la excluía de la solidaridad práctica con otras mujeres, porque al definirse sus tareas como fundamentalmente individuales y relegadas, por ejemplo, a tareas adscritas al rol de amas de casa, hacen que de aquella consideración nazca la superioridad aparente del hombre sobre ella. Este status quo vivendis viene a ser históricamente cuestionado con el surgimiento de círculos sociopolíticos feministas que niegan la actividad del genero femenino como reducido a la cocina y a la crianza de la descendencia. La especificidad y detallismo con que Simmel expone la condición de la mujer diferencia explícitamente entre el movimiento femenino proletario y burgués: «A la mujer proletaria, la evolución industrial le ha dado la libertad económico-social, por miserable que sea su libertad individual. La muchacha va a la fábrica a una edad en que necesitaría la atmósfera estrecha de la casa paterna. A la mujer casada, el trabajo fuera de la casa la ha desligado de sus deberes con el hogar, el marido y los hijos. Por consiguiente: la mujer se encuentra aquí liberada de hecho del vínculo singular que la destinaba a la subordinación al hombre, o, al menos, a una actividad completamente distinta de la del marido" ${ }^{17}$. La situación debe ser necesariamente distinta en la condición social de la burguesía: la misma evolución económica ha anulado, para una cantidad considerable de mujeres, las posibilidades de salir del hogar $\mathrm{y}$, por consiguiente, sus aspiraciones se orientan hacia la libertad del trabajo económico. Estas diferencias cualitativas en la situación concreta de clases da lugar a que algunas mujeres quieran volver a su casa, mientras que otras añoran con salir.

\section{LA SOCIOLOGÍA DE LA MODA}

Una de las formas esenciales y determinantes de la diferenciación social es la moda. El contenido histórico de la estructuración y desestructuración de diferentes modas se apoya en la tendencia inherente del género humano a la imitación, que como herencia psicológica «nos permite actuar de manera adecuada y con sentido aun en los casos en los que no hay nada personal ni creati-

16 Georg Simmel (1968): Das Individuelle Gesetz. Philosophische Exkurse, Suhrkamp, Frankfurt a.M.; Georg Simmel (1986): Sociología II, Alianza, Madrid.

17 Georg Simmel (1986): Sociología II, Alianza, Madrid, p. 469. 
vo por nuestra parte» ${ }^{18}$ (Simmel, 1988: 27). Siendo hija del pensamiento y de la estupidez, la imitación conlleva, por un lado, la seguridad de no sentirse aislado en contextos sociales, proporcionando la tranquilidad necesaria para poner en escena los roles de la identidad que nos asignamos, y además satisface la necesidad de fusión de los sujetos sociales con colectividades procurando mantener, en lo posible, el máximo de individualidad: "la moda es imitación de un modelo dado y proporciona así satisfacción a la necesidad de apoyo social; conduce al individuo al mismo camino por el que todos transitan y facilita una pauta general que hace de la conducta de cada uno un mero ejemplo de ella» ${ }^{19}$.

Las modas son siempre modas de clase, no únicamente porque la moda de las clases dominantes se diferencie cuantitativamente de la de las clases inferiores y sea abandonada en el momento en que esta última comienza a hacerla suya, sino porque la moda es un producto de la división de clases, legitima y sedimenta relaciones de inclusión y exclusión a grupos de «iguales», garantiza la unidad de círculos sociales específicos y otorga sentido desde la exterioridad a círculos sociales diferenciados.

Unir y diferenciar, he ahí las funciones básicas de la moda en las sociedades. Si antaño el origen de modas decía relación con inventos particulares de personalidades, en la modernidad, al margen de perder la moda toda relación con lo estético, ella se subordina a los mecanismos de funcionamiento de la economía: la moda se promueve, se inventa, se diferencia y se especializa pasando a constituir un mecanismo más en la gestión empresarial; por ello la evolución de la moda es insustancial, perenne, liviana y alejada de la significación práctica de las cosas, la moda es una manifestación particular pero abarcante de la frivolidad. Y lo fundamental de ello es, así Simmel, el hecho de que en las sociedades modernas las formas sociales, los juicios estéticos, el vestido y las formas de estilo y expresión de la cultura se encuentran sometidos a las mutaciones de la moda (o a su función de unificación). El movimiento evolutivo de la moda se desarrolla de la siguiente manera: cuando los estratos inferiores se adueñan de sus contenidos, las clases culturalmente hegemónicas, bajo la presión de la necesidad de la distinción, se ven en la necesidad de imponer elementos cualitativamente diferentes de unificación, verificándose el mismo proceso entre los diversos segmentos de las capas sociales superiores. Por otra parte, la generalización de la economía monetaria, al hacer los objetos accesibles mediante la posesión del medio del dinero, acelera las posibilidades de igualdad del estrato inferior con el estrato superior ${ }^{20}$.

18 Georg Simmel (1988): Sobre la Aventura. Ensayos filosóficos, Península, Barcelona, p. 27.

19 Ídem, p. 28.

20 «Por una parte, la moda ilustra el ethos de fasto y dispendio aristocrático, en las antípodas del moderno espíritu burgués consagrado al ahorro, a la previsión, al cálculo; la moda se halla del lado de la irracionalidad de los placeres mundanos y de la superficialidad lúdica, a contracorriente del espíritu de crecimiento y desarrollo del dominio sobre la naturaleza.» Gilles LIPOVETSKY (1990): El imperio de lo efimero. El destino de la moda en las sociedades modernas, Anagrama, Barcelona, p. 35. 
En la vida de las sociedades modernas, el elemento de homogeneización de la moda es particularmente significativo; por una parte, la presión por seguir la moda y evitar la exclusión es enorme, mientras que dicho fenómeno debe contradecir necesariamente el imperativo de la distinción individualista. Pero como el cambio de moda es tan acelerado, la necesidad de correspondencia con lo nuevo probablemente haga que la dicotomía entre homogeneización e individualidad sea más aparente que real: cuanto más nerviosa es una época, con mayor celeridad cambian las modas. Así, por ejemplo, en la Florencia de 1390 no son constatables modas dominantes en el atuendo masculino, pues se carecía de cohesión social en los estratos dominantes, mientras un caso diferente se observa en Venecia, donde la vestimenta negra para toda la población estaba consignada en la ley, para que fuese imposible constatar el reducidísimo número de los nobles. Para que exista moda, deben confluir ambos elementos constitutivos, el de la unificación y el de la diferenciación. Por ello, la esencia de la moda consiste en que sólo una parte de los grupos sociales la ejerce, mientras que el resto está encaminado hacia ella $^{21}$; por ello, cuando ya ha penetrado en todos los estratos sociales, deja de existir y es sustituida por otra moda emergente.

La moda es uno de los elementos más manifiestos de la ambivalencia de la vida moderna; siempre se supone que su existencia tiende a la expansión, pero en el momento en que su ejercicio se generaliza, disminuye en ella el elemento delimitador; "su cuestión no es ser o no ser, sino que ella es simultáneamente ser y no ser, se sitúa siempre en la divisoria de las aguas entre el pasado y el futuro, proporcionándonos así mientras está en su apogeo un sentimiento de presente tan intenso como pocos fenómenos $»^{22}$. Por eso, la moda domina inextricablemente la conciencia social de la vida moderna ya que las grandes convicciones (Lyotard diría los metarrelatos) van perdiendo fuerza paulatinamente. Y si el carácter efímero de la existencia de una moda específica permite visualizar la inmensa relevancia del presente, ello no es sino una expresión indiscutible de la hegemonía de la linealidad temporal, propia de la modernidad: homogeneización e individuación, globalización y particularización de la forma de vida, buisqueda de los elementos comunes y obsesión por la particularidad de lo individual ${ }^{3}$, tales son las propiedades paradógicas de la modernidad que en la moda se manifiestan.

¿Por qué son las mujeres quienes más intensamente siguen la moda? A este interrogante, Simmel responde con la exacerbada debilidad a la que han sido condenadas las mujeres a lo largo de la historia: el débil evita la individuación, busca protección en la aceptación de lo externo a sus particularidades, y sólo si existe sobre el piso de la costumbre aceptada puede conseguir la particularidad individual; la moda ofrece un mimetismo evidente y simultáneamente la posi-

${ }^{21}$ Georg Simmel (1988): Sobre la Aventura. Ensayos filosóficos, Península, Barcelona, p. 35.

22 Ídem, p. 37.

${ }^{23}$ U. BECK (1988): Gegengifte. Die organisierte Unverantwortlichkeit, Frankfurt a.M. 
bilidad de resaltar, mediante el arreglo particular, la belleza de su individualidad. Así, por ejemplo, en Alemania, con posterioridad a la quiebra del colectivismo medieval, se desata en los siglos XIV y XV una verdadera fiebre de individualidad, de la cual, sin embargo, las mujeres permanecen completamente excluidas. Ahora bien, justamente durante este período, el género femenino buscó compensar su segregación con las modas más extravagantes. Al revés en Italia, donde el Renacimiento ofreció a las mujeres las más amplias posibilidades de desarrollo intelectual, siendo, en las capas altas de la población, las libertades de movimiento prácticamente idénticas entre los géneros; Simmel recuerda que en Italia no se tienen noticias de extravagancias y exageraciones en la moda de la época, en una sociedad que estructuralmente garantizaba individuaciones importantes en las mujeres. Por todo lo anterior, no debe entonces sorprender que las mujeres emancipadas de la modernidad, así Simmel, en su intento por conseguir la igualdad de géneros y la supresión de la segregación, subrayen también su independencia respecto de la moda ${ }^{24}$.

Para Simmel, la moda en toda su complejidad y multiplicidad de manifestaciones constituye un elemento dinámico, pero irreductiblemente ambivalente, en las sociedades modernas; por un lado, la frecuente modificación de la moda supone una eminente esclavización del individuo pero es, a su vez, uno de los elementos fundamentales de la libertad política y social, porque precisamente la moda y sus cambios hacen visible que la culminación de una forma de vida coincide con el inicio de la decadencia de una cultura y que sólo los grupos sociales inquietos y curiosos (como es el caso de las clases medias) son capaces de instrumentalizar las fases de crisis y desorientación en el sentido del cambio hacia una expansión de la libertad individual. Ello es evidente en cuanto estratos sociales se apropian de una moda al mismo tiempo que los estratos superiores la abandonan (por razones que ya explicamos): en este período, el precio de las modas desciende y permite a estratos de ingresos modestos participar de ella. Allí aparece un curioso círculo dinámico de intercambio: cuanto más rápido sea el cambio de moda, más baratos han de ser sus objetos, y mientras más baratos sean éstos, más se incita a los consumidores a cambiar de orientación en el consumo respecto de la moda.

Es sabido que Simmel fue quien con espectacular maestría caracteriza a la modernidad como "un incremento de la fragmentación de la vida social y la individual, una fragmentación que requiere un contrapeso. Éste puede hallarse en la adhesión a la moda que confiere a los individuos una cierta supra-individualidad $»^{25}$. Mediante la moda, los individuos parecen contrapesar la indife-

${ }^{24}$ Georg Simmel (1988): Sobre la Aventura. Ensayos filosóficos, Península, Barcelona; David FrISBY (1988): «Georg Simmel: Primer sociólogo de la modernidad», en Josep Picó (ed.) (1988): Modernidad y Posmodernidad, Alianza, Madrid, pp. 51-86.

${ }^{25}$ David FrISBY (1988): «Georg Simmel: Primer sociólogo de la modernidad», en Josep Picó (ed.) (1988): Modernidad y Posmodernidad, Alianza, Madrid, p. 77. De la misma manera que Sombart avalúa el rol del lujo en la constitución y articulación del capitalismo, en W. SOMBART (1988): Lujo y capitalismo, Alianza, Madrid. 
rencia de los acontecimientos contingentes, en un universo respecto del cual se sienten extraños y a la vez participantes. Con la adhesión a la moda, se genera la ilusión de haber alcanzado la dominación sobre las cosas con la propia fantasía falsificada.

\section{CULTURA OBJETIVA Y CULTURA SUBJETIVA}

Si para Simmel la sociedad existe en la medida en que muchos individuos contraen relaciones específicas de intercambio ${ }^{26}$, así, en las diversas manifestaciones históricas que las sociedades asumen, el contenido y la forma de dichas relaciones se encuentran entrelazados de tal manera que ambos no sean sino plausibles de problematizar de la manera siguiente: el contenido de una sociedad o actividad humana puede ser objetivo — como es el caso de la producción de una obra, del progreso de la técnica, de la dominación de una idea, del apogeo o decadencia de un constructo político o del desarrollo del lenguaje o de las formas de integración moral - o bien de naturaleza subjetiva y abarcar las más diversas facetas de la personalidad individual, como la moral, las costumbres y todo lo que tienda a comprender el movimiento del espíritu. De allí que esta relación dialéctica entre individuo y sociedad, entre lo objetivo y lo subjetivo, no pueda ser sino la cultura, concebida como forma; responsable de la construcción de mundos de producciones culturales o verdaderas colecciones de formas, la cultura es la síntesis del contenido de la vida donde confluyen el alma (la fuerza elemental de la actividad humana) y las manifestaciones de la vida que, según Simmel, terminan en la modernidad por alcanzar autonomía respecto de los sujetos sociales que las han producido ${ }^{27}$.

Por ello, la cultura objetiva se desplaza en el ámbito de los instrumentos que los hombres han creado y producido (como las grandiosas formas de vida y organización de las metrópolis de la modernidad), mientras que la subjetiva se ubica en la vida espiritual de la personalidad individual. Simmel, en tal sentido, desechando la posibilidad de dicotomizaciones a priori entre ambas formas de la cultura, ubica históricamente la hegemonía de la cultura objetiva sobre las manifestaciones del alma individual en los albores de la instauración irreversible del capitalismo: cada aumento de la complejidad de la cultura objetiva lleva implicada una inaccesibilidad sistemática desde las subjetividades de los individuos y, en consecuencia, la puesta en marcha de la enajenación creciente de la conciencia individual, arrollada por un mundo de productos objetivos e instrumentales independientes de los espíritus individuales que les han dado origen ${ }^{28}$.

${ }^{26}$ Georg Simmel (1968): Das Individuelle Gesetz. Philosophische Exkurse, Suhrkamp, Frankfurt a.M., p. 43.

${ }^{27}$ Michael Landmann (1968): Einleitung des Herausgeber, en Georg Simmel, ibidem (1968), p. 17.

28 Georg Simmel (1988): Sobre la Aventura. Ensayos filosóficos, Península, Barcelona, pp. 204 y ss. 
Si la obra de Karl Marx acentúa las condiciones estructurales que suministran el contenido y la dirección a la dominación del mundo de las mercancías hasta transformarse en fetiches en la vida social dominada por el dinero y describe la jornada diaria de trabajo " $a$ dos voces» - la del capitalista, que "no es más que el capital personificado. Su alma es el alma del capital», hasta que "de pronto se alza la voz del obrero, que había enmudecido en medio del tráfago del proceso de producción. La mercancía que te he vendido, dice esta voz, se distingue de la chusma de las otras mercancías en que su uso crea valor, más valor del que costó ${ }^{29}$ —, los trabajos de Simmel, partiendo de la autonomización de la cultura objetiva en la modernidad dominada por la división del trabajo y el desarrollo tecnológico, constatan un rezagamiento estructural en el desarrollo cultural de los espíritus individuales frente a la explosión incontrolable de los productos culturales objetivos, resaltando «la tensión constante, el conflicto mismo, entre la energía vital y su manifestación en formas que tienden a adquirir una autonomía que acaba por oprimirla, a pesar de constituirse esas mismas formas en elementos esenciales para las posibilidades de realización de la energía vital» ${ }^{30}$.

Pero si (a) la primera voz, la del capitalista que controla y comercializa los productos de la actividad de interacción y cooperación de los sujetos sociales, sólo puede fantasear con apoderarse también de los efectos de la cultura objetiva y permanece necesariamente sometida a la lógica de un solo instinto vital: el acrecentamiento de la utilidad ${ }^{31}$, (b) la segunda voz, la de los sujetos que dan vida y fuerza a los productos de la cultura objetiva, permanece también sometida al extrañamiento creciente de una lógica inmanente del proceso de división del trabajo y de desarrollo tecnológico: no hay productos objetivos que no se desarrollen al margen de los propósitos originales de los actores y espiritus (cultura subjetiva) que les han dado origen. Y en este mismo sentido, si bien el diagnóstico de Simmel respecto de las consecuencias enajenantes (y perversas, diríamos hoy) de la modernidad es coincidente con los resultados que Marx suministra, Georg Simmel rechaza la funcionalización o "dependencia en última instancia» de las estructuras jurídicas, estéticas, ideológicas o políticas de las formas materiales de producción y reproducción sociales, pues cada una de ellas desencadena procesos de autonomización con contenidos y propiedades específicos, y una vez creadas y sedimentadas estas estructuras, resulta imposible controlar las direcciones y los sentidos que puedan asumir. Dicha lógica elemental del desarrollo de la modernidad es denominada por Simmel la tragedia real de la cultura $^{32}$ (Simmel, 1988: 204-232).

En efecto, la acción instrumental que es propia de la modernidad opera en

29 Karl Marx (1975): El Capital I, FCE, México, 1975, pp. 178-179.

30 Antonio Gil Villegas (1997): «El fundamento filosófico de la teoría de la modernidad en Simmel», en Estudios Sociológicos, XV: 43, México, p. 38.

31 Karl Marx (1975): El Capital I, FCE, México, 1975, p. 179.

32 Georg Simmel (1988): Sobre la Aventura. Ensayos filosóficos, Península, Barcelona, pp. 204-232. 
la práctica de tal manera que los medios orientados hacia fines preestablecidos se transfiguran en fines en sí y, por lo tanto, adoptan una lógica propia e impredecible. El ejemplo prototipo del fenómeno de la metamorfosis de los medios en fines es la función del dinero, que Simmel desarrolla in extenso en su libro Filosofía del dinero: la tergiversación de la existencia (o carencia) de dinero desde su condición de medio orientado a la consecución de fines determinados hasta convertirse en un fin en sí, y por lo tanto excluido de la teleología, pone de manifiesto que las libertades formales del sujeto de la modernidad, mientras estén sujetas a tal proceso de cosificación, no pasan de ser una fantasmagoría más en el contexto de la enajenación sistemática que informa de la pauperización de la cultura subjetiva. Pues al ser formulada la pregunta crucial sobre el sentido del dinero en el capitalismo, los términos de las respuestas resultan ser necesariamente tautológicos. Y esto no tanto porque la voluntad de los espíritus de las sociedades deseen o añoren consciente y deliberadamente que en la vida social reine tal confusión entre medios y fines, que los medios se conviertan en fines y que los fines sean cada vez más difusos, sino porque la creciente complejidad de la vida humana en la modernidad mediatizada por la técnica y la acción instrumental (racional con arreglo a fines, en el sentido de Weber) impone una creciente regresión en el esclarecimiento de sentidos intersubjetivos. En efecto, al desaparecer las cosmovisiones sacrales (como la salvación del alma y el reino de los cielos) de la premodernidad, los sujetos (cultura subjetiva) de la modernidad se ven abandonados a tal secularización de los mundos cotidianos históricamente determinados que su respuesta resulta necesariamente preñada de desorientación y ambivalencia ${ }^{33}$ (Simmel, 1986: 479 y ss.).

Mientras Weber problematiza el fenómeno del Entzauberung der Welt mediante el estudio comparativo de las religiones frente al impacto de la mercantilización y del capitalismo ${ }^{34}$, el enfoque de la filosofía de la vida que Simmel propone enfatiza el dilema del sujeto moderno atrapado en la racionalidad instrumental de la cultura objetiva hegemónica: «la división del trabajo independiza el producto como tal de cada uno de los contribuyentes; el producto está ahí en la objetividad autónoma que, sin duda, lo hace apropiado para acomodarse a un orden de cosas o para servir a un fin particular objetivamente determinado; pero con ello se le escapa aquel estado interno dotado de alma que sólo el hombre en su totalidad puede dar a la obra en su totalidad y que porta su inclusión en la centralidad anímica de otros sujetos» ${ }^{35}$.

¿Cómo contrarrestar los efectos enajenantes de una modernidad donde la cultura objetiva tiende irremediablemente a dominar sin contrapeso sobre la individualidad subjetiva? A través de la obra de Simmel parecieran existir ciertas esferas de las cotidianeidades humanas (cuasi «relaciones-nosotros» en el sentido de Schütz ${ }^{36}$ ) que configuran verdaderas islas independientes de subjetivi-

33 Georg Simmel (1986): Sociología II, Alianza, Madrid, pp. 479 y ss.

${ }^{34}$ M. Weber (1998): Sociología de la religión, Istmo, Madrid, 1997.

35 Georg Simmel (1988): Sobre la Aventura. Ensayos filosóficos, Península, Barcelona, p. 230.

36 A. SCHÜTZ (1995): El problema de la realidad social, Amorrortu, Buenos Aires. 
dad, esferas de la privacidad que compensarían una subjetividad arrasada por la creciente complejidad de la acción instrumental. Precisamente la paradoja de la vida moderna consiste, así Simmel, en que, por liberar a la conciencia de las ataduras de la colectivización premoderna, inaugura simultáneamente espacios originales para el desarrollo de la individualidad. De allí que, a diferencia de Marx y su confianza ciega en la fuerza de transformación de una de las clases en conflicto en el capitalismo, el proletariado, Simmel sostenga que precisamente porque la hegemonía de la cultura objetiva no puede negarse redoblando la homogeneización de los espíritus, sino subrayando su especificidad, la alternativa a una modernidad consista en la búsqueda de formas eminentemente nuevas de interioridad subjetiva ${ }^{37}$.

\section{DE LA AUTONOMIZACIÓN DE LO OBJETIVO A LA INDIVIDUACIÓN DE LAS COTIDIANEIDADES}

Uno de los más connotados biógrafos y analistas del pensamiento de Georg Simmel, David Frisby, apunta que la tematización del fenómeno de la individuación en las sociedades modernas "plantea el problema de hasta qué punto, en tal teoría de la enajenación cultural, el concepto de sociedad desaparece totalmente, o si las sociedades se convierten en sinónimo de la cultura ${ }^{38}$. Ciertamente que si la propuesta de individuación que Simmel formula se interpreta en calidad de retirada existencialista al reino del autismo grupal «alternativo» a un mundo empapado de cosificación, la doble enajenación que ello encierra debiese ser obligadamente juzgada como una evidente negación de lo social ${ }^{39}$. No obstante, a mi juicio, las argumentaciones de Simmel van mucho más allá de esta hermenéutica algo simplista de sus ensayos abriendo paso a una visión tanto original como precisa de la modernidad, argumento que voy a exponer en dos pasos consecutivos:

1. Si la cúspide de la hegemonía de la cultura objetiva sobre la subjetividad individual se pone de manifiesto en la vida social de la metrópoli contemporánea ${ }^{40}$ con su avasalladora urbanización, colectivización y anonimización del mundo de los significados, no sólo resultaría metodológicamente contradictorio, sino eminentemente tautológico re-caer en la trampa de la reflexión instrumental (que caracteriza a la dominación de la cultura objetiva) para destinar esfuerzos de comprensión a la estructuración «objetiva» de elementos y estruc-

37 David Frisby (1988): "Georg Simmel: Primer sociólogo de la modernidad», en Josep Picó (ed.) (1988): Modernidad y Posmodernidad, Alianza, Madrid, p. 81.

38 Ídem, p. 110.

39 Theodor W. Adorno (1966): Kierkegaard, Suhrkamp, Frankfurt a. M.

${ }^{40}$ Georg Simmel (1903): «Soziologie des Raumes», en Jahrbuch für Gesetzgebung, Verwaltung und Volkswirtschaft, 27, pp. 27-71. 
turas que supuestamente «determinarían» dicha dominación con todas sus consecuencias enajenantes. Porque si hacemos arrancar inseparablemente cualquier reflexión sobre las condiciones de constitución del sentido y del mundo desde los significados de la interacción existente entre sujetos concretos en la vida moderna, no queda otra alternativa que remitirse a la descripción de dichas condiciones. Y si Marx realiza tal empresa distinguiendo entre la subsunción formal y subsunción real del trabajador frente a la inminencia de su situación en el mundo ${ }^{41}$, Simmel se dispone a examinar las estrategias de autopreservación biográfica que los individuos habitantes de la ciudad moderna despliegan para enfrentarse a una masificación anonimizante de un mundo que se dispone a transitar sin considerar sus sentimientos, sus precariedades, sus angustias y sus necesidades.

Todo este complejo y esforzado proceso de recomposición adaptativa de las biografías individuales en medios (Millieu) donde los sujetos se transforman en extraños a sí mismos y a sus semejantes y se resisten a ser nivelados y devastados por el medio sociotecnológico, se sitúa en el centro de la sociología de Simmel. De allí que resulte no sólo equivocado, sino altamente injusto, confundir la resultante de individuación creciente que Simmel constata como contrapartida al creciente ordenamiento objetivizante de la vida en la modernidad, sencillamente con supuestas petitio principi a favor de una subjetivización en retirada que se traduciría en la reificación de la privacidad. El exagerado subjetivismo que Simmel diagnostica en calidad de contrapuntos múltiples a la nivelación o colectivización de la cotidianeidad que impone la metrópoli de la modernidad como prolongación implícita de la economía monetaria, resulta ser la única vía factible de recomposición de la subjetividad y, en tal sentido, la postura de Simmel inaugura las constataciones de indiferencia y hedonización del mundo que, en la contemporaneidad de fines de siglo, forman parte de los factores más altamente aceptados de la modernidad postindustrial ${ }^{42}$.

2. La indiferencia y el hastío del hombre contemporáneo y la consiguiente estetización del mundo a la búsqueda de lo bueno y lo bello en la vida, tanto como la reserva y la desconfianza entre inclusive los miembros de estratos sociales unificados por círculos sociales relativamente similares, son mecanismos de defensa frente a empresas de colectivización cuyo desenlace permanece, para quienes de ellas participan, sistemáticamente en la oscuridad. Por ello, Simmel utiliza la metáfora del laberinto, una y otra vez para ilustrar el camino incierto de las subjetividades de la modernidad vertebradas en cotidianeidades múltiples, incesantes e impredecibles, de facto unificadas por las economías del dinero, que «no simboliza el mero movimiento dentro de la sociedad concebi-

${ }^{41}$ Karl MarX (1990): El capital. Libro I, Capitulo VI. Resultados del proceso inmediato de producción, Siglo XXI, México.

42 Daniel Bell (1977): Las contradicciones culturales del capitalismo, Alianza, Madrid; Scott LASH y John URRY (1998): Economias de signos y espacios. Sobre el capitalismo de la posorganización, Amorrortu, Buenos Aires. 
da como un laberinto; su función dentro del intercambio también crea todas las conexiones que constituyen el laberinto económico. Es la araña que teje la tela de la sociedad $»^{43}$.

A Simmel no le interesan las teorías de la producción que procuren explicar los mecanismos sistémicos de generación y distribución de la riqueza, sino las consecuencias individuales del intercambio mercantil en el centro de las sociedades modernas considerando (a) que el intercambio es un mecanismo crucial de la socialización de los seres humanos y (b) que dicho intercambio en las sociedades modernas está empapado por el mundo de las mercancías y del dinero, punto en el que, evidentemente, coincide con Marx. Pero si uno de los mecanismos indispensables para el funcionamiento de las economías del dinero es justamente la fragmentación de las individualidades y la transformación de los sujetos en consumidores de mercancías, Simmel, a diferencia de Marx, que diagnostica la exacerbación de las luchas entre las clases acompañada de la pauperización inminente del proletariado, tiene en vista una respuesta simultáneamente efectiva pero imperceptible a la disociación de las subjetividades y a la fragmentación de los individuos. En efecto, quienes sean excluidos del mercado y del consumo no pugnarán por transformar las condiciones estructurales bajo las cuales han sido excluidos para excluir (expropiar) a los culpables de dicha segregación, sino que procurarán crear las condiciones para permanecer establemente incluidos y participar de la moda, del estilo, de las cotidianeidades que le confieran el «toque de distinción» sobre sus semejantes; este proceso, designado con el nombre de "objetivación del estilo de vida» en el período moderno, hace que los conflictos entre formas de vida, entre las culturas, subculturas y contraculturas, entre individualidades que componen el sentido de una u otra manera, se superpongan a las luchas sociales que pueden ser estructuralmente motivadas. Como apunta correctamente la obra de Scott Lash, dicha "economía de signo y espacio» inaugura una nueva subjetividad reflexiva, la que, muy lejos de arrojar a los «excluidos» por el sendero de la anomia (Durkheim), se plasma en la existencia de "comunidades reflexivas»: «Estas comunidades son reflexivas en los siguientes respectos: primero, uno no nace o "es arrojado" a ellas, sino que "se arroja a sí mismo"; segundo, pueden extenderse ampliamente a través de un "espacio abstracto", y quizá también a través del tiempo; tercero, se plantean conscientemente el problema de su propia creación y constante reinvención mucho más de lo que lo hacen las comunidades tradicionales; cuarto, sus "herramientas" y productos tienden a ser no materiales, sino abstractas y culturales $»^{44}$.

Corresponde, no obstante, formular las siguientes interrogantes: ¿Cuál debiera ser la postura práctica de los desencantados del desencantamiento de los

43 David Frisby (1988): "Georg Simmel: Primer sociólogo de la modernidad», en Josep Picó (ed.) (1988): Modernidad y Posmodernidad, Alianza, Madrid, p. 70.

44 S. LASH (1997): «La reflexividad y sus dobles: estructura, estética y comunidad», en U. Beck, A. Giddens y S. Lash (eds.) (1997), p. 200. 
proyectos de transformación estructural de las sociedades que, imposibilitados de excluirse absolutamente del consumo y del dinero, sin embargo, se niegan a aceptar una inclusión idiotizante a la modernidad? ¿Los nuevos movimientos sociales, como los homosexuales, los extranjeros inmigrantes, los desocupados, los nuevos pobres, los ecologistas, no constituyen acaso contrapartidas multifacéticas e individualizadas frente a "consecuencias perversas ${ }^{45}$ e imprevistas a una modernidad que ha convertido en un dogma la racionalidad instrumental de la cultura objetiva? Procurando hacer justicia a las ambigüedades e imprecisiones ensayísticas de la obra de Simmel, se debe concluir necesariamente que sus investigaciones inauguran análisis hoy considerados decisivos para la comprensión de la modernidad.

\section{ABSTRACT}

This papper outlines several features of modern societies which are shaped by the sociological category of ambivalences, thus, the objetive culture and the subjective culture, the mode, the collectivization and the individualization. The main basic assumption is the position of the "AND» instead of the position "EITHER/OR».

45 Josetxo Beriain (1996): «El doble "sentido" de las consecuencias perversas de la modernidad», en J. Beriain (comp.): Las consecuencias perversas de la modernidad, Anthropos, Barcelona, pp. $7-30$. 